\title{
Functional brain imaging applications to differential diagnosis in the dementias \\ Eric Salmon
}

\begin{abstract}
Purpose of review
The diagnosis of dementia rests on an improved knowledge and a better detection of early impairments, to which functional imaging can certainly contribute.

\section{Recent findings}

Progress has been observed at different levels. First, the understanding of different dementias has benefited from explorations of the neural substrate of dementia symptoms and from research into new markers. Second, diverse variables (clinical, anatomical, biochemical) have been related to impaired cerebral activity in Alzheimer's disease and other dementias, and progress in image analysis and in multimodal data acquisition has allowed a better understanding of the significance of brain activity disturbances. Third, functional imaging has been applied in well-designed clinical studies, and has provided important arguments for the diagnosis of characteristic clinical syndromes in the dementias.

\section{Summary}

The functioning of neural networks responsible for clinical symptoms in dementia remains an important research topic for functional imaging. The development of new tracers and new techniques for image processing should also improve the usefulness of brain imaging as a diagnostic tool.
\end{abstract}

\section{Keywords}

dementia, imaging

Curr Opin Neurol 15:439-444. (C) 2002 Lippincott Williams \& Wilkins.

Department of Neurology and Cyclotron Research Centre, University of Liege, Liege, Belgium

Correspondence to Professor Eric Salmon, Cyclotron Research Centre, University of Liege, B30 Sart Tilman, 4000 Liege, Belgium

Tel: +32 4 3662316; fax: +32 4 3662946; e-mail: eric.salmon@ulg.ac.be

Current Opinion in Neurology 2002, 15:439-444

Abbreviations

$\begin{array}{ll}\text { AchE } & \text { acetylcholine esterase } \\ \text { AD } & \text { Alzheimer's disease } \\ \text { CBF } & \text { cerebral blood flow } \\ \text { DLB } & \text { dementia with Lewy bodies } \\ \text { FDG } & \text { 2-[(18)F]fluoro-2-deoxy-D-glucose } \\ \text { FTD } & \text { frontotemporal dementia } \\ \text { MCI } & \text { mild cognitive impairment without dementia } \\ \text { MRI } & \text { magnetic resonance imaging } \\ \text { MTR } & \text { magnetization transfer ratio } \\ \text { PET } & \text { positron emission tomography }\end{array}$

(C) 2002 Lippincott Williams \& Wilkins $1350-7540$

\section{Introduction}

Functional imaging is an essential tool to study the neural substrate and the physiopathology of clinical syndromes in different dementias. That research will constitute the main purpose of this article. When functional disturbances are sufficiently characteristic, functional imaging may be used for the differential diagnosis of dementias. Assessing the relative sensitivity and specificity, and eventually the advantage of different techniques for reaching a specific diagnosis remains difficult because neuropathological confirmation is rare and a between-study comparison of percentages is not fair, whereas a within-study comparison of different functional imaging techniques is infrequent. In practice, there is a trade-off in functional imaging between, for example, ongoing refinements in image analysis, the availability of the techniques and the feasibility of studies in clinical versus research settings.

\section{Diagnostic value of functional imaging in Alzheimer's disease}

Different variables need to be considered when evaluating the diagnostic value of functional imaging, such as the size of the population, the probability of the diagnoses, the technique and the method for image analysis (for example, the pattern considered to be characteristic of Alzheimer's disease; AD). When compared with a clinical diagnosis, the visual analysis of single-photon emission computed tomography images has been shown to increase the likelihood of a neuropathological diagnosis of $\mathrm{AD}$ [ $1^{\bullet}$. In a large population of 138 cases with different neuropathologically confirmed diagnoses, the visual analysis of positron emission tomography (PET) images identified patients with $\mathrm{AD}$ and patients with any neurodegenerative disease, with a sensitivity of $94 \%$ and specificities of $73 \%$ and $78 \%$, respectively [2॰]. Those results were confirmed for questionable or mild AD. In that study, a 'non-characteristic' PET image predicted that subsequent progression to definite dementia occurred in only $30 \%$ of the cases. Other methods for image analysis will be reviewed in the following paragraphs. They might improve both the sensitivity and specificity of functional imaging for the diagnosis of dementia, but implementation in the clinical environment would require more resources. 


\section{The meaning of the regional brain distribution of functional activity in Alzheimer's disease}

AD has been characterized for a long time by a metabolic decrease predominant in the posterior associative cortices. Before concentrating on $\mathrm{AD}$, it is important to underline that the distribution of cerebral activity observed with functional imaging is not unique to $\mathrm{AD}$ pathology, as demonstrated by values of specificity in diagnostic studies and by data on non-Alzheimer dementias discussed in subsequent paragraphs. For example, a reversible 'AD compatible' decrease of perfusion in the posterior associative cortices was observed in hyperthyroid dementia, emphasizing the essential relationship between the clinical syndrome and functional imaging [3].

\section{Evolution of cerebral functional activity in Alzheimer's disease}

In the early stages of $\mathrm{AD}$, some authors have suggested that hypoperfusion would start in the medial temporal regions, where lowered cerebral blood flow (CBF) might subsequently reach a plateau [4]. Activity in the lateral temporoparietal (and medial posterior) associative cortices would progressively decrease with the severity of dementia, and would become more important than impaired activity in the hippocampal areas, producing the typical pattern reported in most studies, whereas functional impairment would occur later in the prefrontal regions [5]. However, the magnitude and the sequence of longitudinal CBF changes may vary between different $\mathrm{AD}$ populations or according to different analysis procedures [6]. In keeping with the heterogeneity of $\mathrm{AD}$, the selective impairment of episodic and semantic memory in a subgroup of patients was associated with hypoperfusion in the bilateral mesial temporal regions of interest, whereas the posterior associative cortices were predominantly involved in AD patients with more widespread cognitive impairment $\left[7^{\bullet}\right]$.

In a study of carriers of the PS- 1 mutation [ $8^{\bullet}$ ], regional cerebral perfusion abnormalities were detected in the hippocampal complex and in the posterior associative cortices before the development of the clinical symptoms of AD. A metabolic pattern similar to that observed in $\mathrm{AD}$ was previously reported in cognitively normal carriers of the apolipoprotein E epsilon4 allele with a familial history of AD [9]. Those patients were followed over an interval of approximately 2 years, and they suffered a significant decline in glucose metabolism in the vicinity of the parahippocampal gyrus, temporal, posterior cingulate and the prefrontal cortices, basal forebrain and thalamus $\left[10^{\bullet}\right]$. The authors emphasized the availability of PET to test the efficacy of treatments for attenuating this functional decline.
Changes in brain activation in Alzheimer's disease

An impaired odour discrimination is reported in AD, and a decrease in right piriform and anterior ventral temporal activation was observed during olfactory stimulation when patients were compared with elderly controls [11]. Brain activation was studied for forced choice face recognition, and the authors demonstrated that different functional networks are activated for similar tasks in AD and in elderly controls [12॰]. AD patients presented increases or decreases of activation compared with control subjects. Cerebral activation in $\mathrm{AD}$ remains to be explained in terms of performance and strategy during cognitive tasks. Moreover, the relationship between brain activation and atrophy is a complex one, for increased activation has been related to the loss of cortical gray matter, suggesting a possible compensation in some (but not all) brain regions in AD [13].

\section{Relationships between brain activity and clinical variables}

The degree of hypoperfusion in the temporoparietal areas of demented patients at the first visit has been shown to predict the achievement of endpoints such as loss of activity in daily living, incontinence and death as

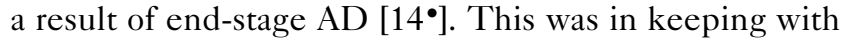
a previous study [15], which showed that temporal metabolism in questionable AD predicted the evolution to a probable diagnosis of this dementia. Several questions are not yet settled, such as the influence of demographic variables (premorbid intelligence, for example) on the level of metabolism observed at a given stage of dementia [16].

AD, with its clinical heterogeneity, remains a particularly interesting condition to study clinical and metabolic relationships. Retrieval in verbal episodic memory has been related to left entorhinal activity in AD [17], whereas verbal semantic memory tests in AD patients have been correlated to glucose metabolism in the left inferior temporal gyrus [18]. AD patients with apathy were shown to have anterior cingulate hypoperfusion compared with patients without apathy $\left[1^{\bullet}\right]$. Clinical-metabolic correlations have essentially been studied in transversal studies, using either a-priori hypothesized regions of interest or (more convincingly) voxel-based statistical analysis, which takes the entire brain volume of activity into account. However, reports on the sensitivity of functional imaging show that few demented patients may have normal activity distribution, whereas abnormal metabolism was observed in asymptomatic at-risk subjects. More sensitive methods for image analyses and longitudinal studies are required to explore further the multifactorial clinical-metabolic relationships. 
Relationship between functional and anatomical data in Alzheimer's disease

The characteristic functional involvement of the temporoparietal and posterior cingulate cortices in presymptomatic and clinically demented $\mathrm{AD}$ patients has been reproduced by both longitudinal and cross-sectional voxel-based morphometry studies using magnetic resonance imaging (MRI), which have also demonstrated medial temporal atrophy $\left[20,21^{\circ}\right]$. Possible artefacts introduced by stereotactic anatomical standardization of atrophied brains have been discussed when voxel-based statistical mapping methods are used to analyse brain imaging in patients [22], but automated techniques are capable of facilitating the serial measurement of medial temporal volumetric changes in $\mathrm{AD}\left[2^{\circ}\right]$. New computational approaches distinguishing variations in gray matter distribution from variations in gyral patterns have shown that the greatest deficits in gray matter loss were mapped in the temporoparietal cortices [24 ${ }^{\bullet}$. Degeneration within the prefrontal cortex appeared to be most prominent in the inferior gyrus in patients with $\mathrm{AD}[25]$.

Studies that combined functional and anatomical imaging have shown that between-group comparisons of regional metabolic values are diagnostically superior to volume measurements $\left[26^{\circ}\right.$, and that atrophy is not sufficient to explain the reduction of brain activity in AD $[27,28]$. However, conflicting results exist for the medial temporal region, which might depend on differential pathophysiology, sensitivity or susceptibility to artefacts of functional and volumetric measurements [29]. Regional perfusion and brain metabolism are correlated in $\mathrm{AD}$, but there remain many anatomical variables that might account for reduced cerebral activity in the disease. A decrease in metabolic activity was suggested to be related to reduced synaptic activity more than to neuronal loss [30]. A decrement of cytochrome oxidase histochemistry, used as an intracellular measure of oxidative energy metabolic capacity, has been shown in the posterior cingulate cortex of $\mathrm{AD}$ patients, and might contribute to the metabolic impairment observed in functional imaging [31].

\section{Functional magnetic imaging techniques in Alzheimer's disease}

When we consider the number of biochemical, anatomical, demographic or clinical variables that may be related to brain functional activity in $\mathrm{AD}$, it remains important to explore new functional parameters to improve our understanding of the physiopathology of the disease. MRI of the regional cerebral blood volume has shown decreased values in the cortical and hippocampal regions of AD patients, even when atrophy was used as a covariate [32]. An apparent coefficient of water diffusion was not statistically different between $\mathrm{AD}$ and controls in that study. However, it was found to be relatively increased in the temporal stem and posterior cingulate, the occipital, and parietal white matter in another population of $\mathrm{AD}$ patients compared with control subjects [33]. Another study [34] demonstrated a reduced magnetization transfer ratio (MTR) and increased mean diffusivity in the cortical gray matter of $\mathrm{AD}$ patients compared with control subjects. A decrease in the MTR has been found in the hippocampus of $\mathrm{AD}$ patients with very mild dementia [35]. A low MTR ratio indicates a reduced capacity of the macromolecules in tissue membranes of the brain to exchange magnetization with the surrounding water molecules, reflecting damage to these membranes. Increased water diffusivity reflects a disintegration of brain tissue compartments. Further studies are required to correct the values for brain atrophy and to specify their meaning. The measurements with ${ }^{1} \mathrm{H}$-magnetic resonance spectroscopy of absolute metabolite concentrations in the associative neocortex showed abnormal results in $\mathrm{AD}$. Abnormalities of $N$-acetyl aspartate were disputed in mild $\mathrm{AD}$, but they were positively correlated to the severity of dementia $[36,37]$. This probably reflects decreased neuronal viability in $\mathrm{AD}$. A weak, but significant positive correlation was observed between regional cerebral glucose metabolism measured using 2-[(18)F]fluoro-2-deoxy-Dglucose (FDG)-PET and $N$-acetyl aspartate relative signal intensity in $\mathrm{AD}$ patients [38 $8^{\circ}$. Diffusion tensor imaging showed a highly significant reduction in the integrity of the association white matter fibre tracts in the splenium of the corpus callosum, superior longitudinal fasciculus, and cingulum in patients with probable $\mathrm{AD}$ compared with normal controls [39].

\section{Assessment of physiopathological hypotheses in Alzheimer's disease using functional imaging}

The clinical benefit observed in AD with acetylcholine esterase (AchE) inhibitor therapy has been associated with an increase of metabolism and perfusion in the medial temporal and cortical associative cortices [40-42]. Radiolabelled substrates allow the measurement of cerebral AchE activity with PET [43 ${ }^{\circ}$, and cortical activity is reduced in $\mathrm{AD}$ patients compared with controls [44. $]$. AchE activity was proved to be reduced in vivo in AD patients taking AchE inhibitor treatment [45].

Activated microglia have been detected in vivo in the entorhinal, temporoparietal and cingulate cortex of $\mathrm{AD}$ patients using a specific ligand of the peripheral benzodiazepine binding sites, illustrating the brain's immune response to neuronal degeneration [46 $\left.{ }^{\circ}\right]$. Detection of amyloid plaque burden in vivo in the brain of patients with $\mathrm{AD}$ is also an aim of functional imaging.

\section{Mild cognitive impairment}

MRI volume sampling with co-registered and atrophy corrected FDG-PET scans were used to demonstrate that entorhinal cortex glucose metabolism and hippocampal 
volume were the most accurate variables to distinguish mild cognitive impairment without dementia (MCI) from controls [26 $]$. Both imaging modalities identified the temporal neocortex as best separating MCI and AD. Widespread changes accurately classified AD and controls. In most between-group comparisons, regional metabolic values were diagnostically superior to volume measurements. In keeping with those results, a measure of temporoparietal cerebral metabolism was shown to predict the evolution of patients with MCI to AD [47]. A regional decrease in perfusion in the hippocampal-amygdaloid complex, the posterior cingulate, the anterior thalamus, and the anterior cingulate had already been shown to be most prominent among MCI patients who subsequently converted to AD [48]. MRI has been used to show that the hippocampal-apparent diffusion coefficient was higher in MCI and AD patients than in control subjects [33].

The predictive value of FDG-PET was further demonstrated in a longitudinal study of initially normal elderly control subjects [49 • $]$. Subjects declining at follow-up and non-declining controls were matched for demographic variables. Glucose metabolism in the entorhinal cortex was shown to predict cognitive decline to MCI or even to AD. Among those who declined, the baseline entorhinal cortex metabolism predicted longitudinal memory and temporal neocortex metabolic impairments.

\section{Vascular dementia}

Global cognitive impairment has been related to both whole cortical and frontal hypoperfusion in vascular dementia. Frontal lobe perfusion was associated with subcortical hyperintensity volume measured on MRI, but not with performances on executive functions [50]. This is in keeping with the idea that executive and attentional functions would depend on distributed (but specific) cerebral networks.

\section{Dementia with Lewy bodies}

A study of autopsy-confirmed AD and patients with dementia with Lewy bodies (DLB) [51 ${ }^{\circ}$ ] has shown a significant metabolic reduction involving the parietal, temporal, posterior cingulate and frontal association cortices, in both conditions compared with control subjects. Only DLB patients showed metabolic impairment in the occipital cortex. Clinically diagnosed probable $\mathrm{AD}$ patients showed a significantly higher frequency of primary visual metabolic reduction among those who later fulfilled clinical criteria for DLB. Therefore, a decrease in occipital activity was proposed as a potential antemortem marker to distinguish DLB from $\mathrm{AD}\left[5^{\bullet}\right]$. However, occipital hypometabolism is not universal in DLB [53].

Occipital glucose metabolism was observed in DLB with and without parkinsonism, compared with $\mathrm{AD}$ patients [54].
Defects in nigrostriatal dopamine pathways in DLB have been demonstrated with functional neuroimaging using ligands probing pre- and post-synaptic dopaminergic systems [55-57]. On the other hand, DLB patients appeared to have less frequent medial temporal functional impairment than an AD group [58]. Accordingly, hippocampal and parahippocampal volumes have been shown to be significantly larger in individuals with DLB compared with AD patients [59]. Caution is mandatory when FDG-PET is used for the differential diagnosis of parkinsonian syndromes with dementia, for hypometabolism in associative cortices and in the caudate has been reported in advanced non-demented patients with Parkinson's disease [60].

\section{Frontotemporal dementia}

A decrease of activity in the frontal and temporal lobes has been confirmed in different cases of frontotemporal dementia (FTD) [61]. Frontotemporal hypoperfusion has been reported in patients with bulbar onset motor neuron disease when they present with cognitive impairment [62]. In keeping with the heterogeneity of the syndrome, dementia in schizophrenic patients has been characterized by memory and executive dysfunction and by lowered frontotemporal CBF [63]. Hypoperfusion in the frontal, anterior cingulate and temporal cortex and in the caudate nucleus has been shown on mean single-photon emission computed tomography images of patients with FTD standardized in a common stereotactical space $\left[6^{\circ}\right]$, as already reported for PET data [65]. The neural substratum of the heterogeneity of FTD has not been much explored by functional imaging [66]. In the domain of behavioural neurology, dramatic changes of self, defined by essential changes in previous social values, have been related to prevalent nondominant frontal hypoperfusion in FTD patients [67]. The density of degenerating astrocytes was inversely correlated to CBF in FTD, but relationships with other lesions were not reported [68].

\section{Miscellaneous dementias}

Although there is no consistent pattern of CBF changes that characterize Creutzfeldt-Jakob disease, a case study of the Heidenhain variant has illustrated the pathophysiological interest of functional imaging. MRI appeared normal in the patient, but his profound visual impairment was accompanied by a marked decrease of perfusion in the primary and associative visual cortices [69].

A study in a population of patients with the clinical phenotype of classical corticobasal degeneration confirmed a characteristic pattern of asymmetrical metabolic impairment in the perirolandic cortical areas and in the central grey nuclei $\left[70^{\bullet}\right]$. Not all patients were impaired when performing gestures on verbal command or to imitation. Those who performed more poorly than elderly volunteers had a significant decrease of metabo- 
lism in the anterior cingulate cortex, suggesting that a global attentional factor was involved. When apraxia was defined as an inability to correct in a second trial erroneous gesture performance, only $38 \%$ of patients with corticobasal degeneration were shown to have a visuo-imitative upper limb apraxia related to metabolic impairment in a superior parietal-frontal network.

\section{Conclusion}

Functional imaging certainly provides useful information in the differential diagnosis of dementia. However, the sensitivity of current studies is not maximal and specificity is relatively poor. Technical progress in image analysis, such as whole-brain voxel-based analyses, multi-modal coregistration in brain atlases, or anatomically driven metabolic measurements, can provide more precise information. But refined pathophysiological research is of the greatest importance to explore further the influence of multiple clinical and biological variables on brain activity measurements. There is also an ongoing search for imaging in vivo more specific brain markers in different diseases.

\section{Acknowledgements}

Work in the Cyclotron Research Centre is funded by the University of Liege, the University Hospital in Liege, the 'Fonds National de la Recherche Scientifique', the 'Fondation Medicale Reine Elisabeth de Belgique', an Interuniversity Attraction Pole, and a European program 'Network for Efficiency and Standardisation of dementia diagnosis'.

\section{References and recommended reading}

Papers of particular interest, published within the annual period of review, have been highlighted as:

- of special interest

-. of outstanding interest

1 Jagust W, Thisted R, Devous MD Sr, et al. SPECT perfusion imaging in the - diagnosis of Alzheimer's disease: a clinical-pathologic study. Neurology 2001; 56:950-956.

A demonstration of the interest of functional imaging for the diagnosis of definite AD.

2 Silverman DH, Small GW, Chang CY, et al. Positron emission tomography in - evaluation of dementia: Regional brain metabolism and long-term outcome. JAMA 2001; 286:2120-2127.

The specificity and sensitivity of functional imaging for the diagnosis of a large series of autopsy confirmed cases of dementia is shown in this study.

3 Fukui T, Hasegawa Y, Takenaka H. Hyperthyroid dementia: clinicoradiological findings and response to treatment. J Neurol Sci 2001; 184:81-88.

4 Nebu A, Ikeda M, Fukuhara R, et al. Utility of (99m)Tc-HM-PAO SPECT hippocampal image to diagnose early stages of Alzheimer's disease using semiquantitative analysis. Dement Geriatr Cogn Disord 2001; 12:153-157.

5 Nebu A, Ikeda M, Fukuhara R, et al. Relationship between blood flow kinetics and severity of Alzheimer's disease: assessment of severity using a questionnaire-type examination, Alzheimer's disease assessment scale, cognitive sub-scale (ADAS(cog)). Dement Geriatr Cogn Disord 2001; 12:318-325.

6 Kogure $D$, Matsuda $H$, Ohnishi $T$, et al. Longitudinal evaluation of early Alzheimer's disease using brain perfusion SPECT. J Nucl Med 2000; 41:1155-1162.

7 Cappa A, Calcagni ML, Villa $G$, et al. Brain perfusion abnormalities in - Alzheimer's disease: comparison between patients with focal temporal lobe dysfunction and patients with diffuse cognitive impairment. J Neurol Neurosurg Psychiatry 2001; 70:22-27.

Heterogeneity in $A D$ is demonstrated by clinical symptoms and brain perfusion.
8 Johnson KA, Lopera F, Jones K, et al. Presenilin-1-associated abnormalities in - regional cerebral perfusion. Neurology 2001; 56:1545-1551.

This study shows the preclinical impairment of brain activity in subjects at risk of AD.

9 Reiman EM, Caselli RJ, Yun LS, et al. Preclinical evidence of Alzheimer's disease in persons homozygous for the epsilon 4 allele for apolipoprotein $\mathrm{E}$. N Engl J Med 1996; 334:752-758.

10 Reiman EM, Caselli RJ, Chen K, et al. Declining brain activity in cognitively

- normal apolipoprotein E epsilon 4 heterozygotes: a foundation for using positron emission tomography to efficiently test treatments to prevent Alzheimer's disease. Proc Natl Acad Sci U S A 2001; 98:3334-3339.

A longitudinal study of brain activity in AD.

11 Kareken DA, Doty RL, Moberg PJ, et al. Olfactory-evoked regional cerebral blood flow in Alzheimer's disease. Neuropsychology 2001; 15:18-29.

12 Grady CL, Furey ML, Pietrini $P$, et al. Altered brain functional connectivity and - impaired short-term memory in Alzheimer's disease. Brain 2001; 124:739-756. This study shows the activation of different brain networks for similar tasks in $A D$ and controls.

13 Johnson SC, Saykin AJ, Baxter LC, et al. The relationship between fMRI activation and cerebral atrophy: comparison of normal aging and Alzheimer disease. Neurolmage 2000; 11:179-187.

14 Nobili F, Copello F, Buffoni F, et al. Regional cerebral blood flow and prognostic evaluation in Alzheimer's disease. Dement Geriatr Cogn Disord 2001; 12:8997.

The level of brain activity predicts the evolution of dementia

15 Herholz K, Nordberg A, Salmon E, et al. Impairment of neocortical metabolism predicts progression in Alzheimer's disease. Dement Geriatr Cogn Disord 1999; 10:494-504.

16 Alexander GE, Furey ML, Grady CL, et al. Association of premorbid intellectual function with cerebral metabolism in Alzheimer's disease: implications for the cognitive reserve hypothesis. Am J Psychiatry 1997; 154:165-172.

17 Eustache F, Desgranges B, Giffard B, et al. Entorhinal cortex disruption causes memory deficit in early Alzheimer's disease as shown by PET. Neuroreport 2001; 12:683-685.

18 Hirono N, Mori E, Ishii K, et al. Neuronal substrates for semantic memory: a positron emission tomography study in Alzheimer's disease. Dement Geriatr Cogn Disord 2001; 12:15-21.

19 Migneco O, Benoit M, Koulibaly PM, et al. Perfusion brain SPECT and statistical

- parametric mapping analysis indicate that apathy is a cingulate syndrome: a study in Alzheimer's disease and nondemented patients. Neurolmage 2001; 13:896-902.

A study of the neural substrate of a behavioural abnormality in AD.

20 Fox NC, Crum WR, Scahill Rl, et al. Imaging of onset and progression of Alzheimer's disease with voxel-compression mapping of serial magnetic resonance images. Lancet 2001; 358:201-205.

21 Baron JC, Chetelat $\mathrm{G}$, Desgranges $\mathrm{B}$, et al. In vivo mapping of gray matter loss with voxel-based morphometry in mild Alzheimer's disease. Neurolmage 2001; 14:298-309.

A well discussed assessment of cortical atrophy using anatomical imaging in AD.

22 Ishii K, Willoch F, Minoshima S, et al. Statistical brain mapping of 18F-FDG PET in Alzheimer's disease: validation of anatomic standardization for atrophied brains. J Nucl Med 2001; 42:548-557.

23 Crum WR, Scahill RI, Fox NC. Automated hippocampal segmentation by

regional fluid registration of serial MRI: validation and application in Alzheimer's disease. Neurolmage 2001; 13:847-855.

A longitudinal assessment of brain atrophy in $A D$.

24 Thompson PM, Mega MS, Woods RP, et al. Cortical change in Alzheimer's

- disease detected with a disease-specific population-based brain atlas. Cereb Cortex $2001 ; 11: 1-16$.

A mapping of different cerebral anatomical characteristics in $A D$.

25 Salat $\mathrm{DH}$, Kaye JA, Janowsky JS. Selective preservation and degeneration within the prefrontal cortex in aging and Alzheimer disease. Arch Neurol 2001; 58:1403-1408.

26 De Santi S, de Leon MJ, Rusinek $\mathrm{H}$, et al. Hippocampal formation glucose

metabolism and volume losses in MCl and AD. Neurobiol Aging 2001; 22:529539

An interesting study combining impaired activity and atrophy in the medial temporal lobe.

27 Ibanez V, Pietrini P, Alexander GE, et al. Regional glucose metabolic abnormalities are not the result of atrophy in Alzheimer's disease. Neurology 1998; 50:1585-1593. 
28 Bokde AL, Pietrini $\mathrm{P}$, Ibanez V, et al. The effect of brain atrophy on cerebral hypometabolism in the visual variant of Alzheimer disease. Arch Neurol 2001; 58:480-486.

29 Kitayama N, Matsuda $\mathrm{H}$, Ohnishi T, et al. Measurements of both hippocampal blood flow and hippocampal gray matter volume in the same individuals with Alzheimer's disease. Nucl Med Commun 2001; 22:473-477.

30 Salmon E, Gregoire MC, Delfiore G, et al. Combined study of cerebral glucose metabolism and [11C]methionine accumulation in probable Alzheimer's disease using positron emission tomography. J Cereb Blood Flow Metab 1996; 16:399-408.

31 Valla J, Berndt JD, Gonzalez-Lima F. Energy hypometabolism in posterior cingulate cortex of Alzheimer's patients: superficial laminar cytochrome oxidase associated with disease duration. J Neurosci 2001; 21:4923-4930.

32 Bozzao A, Floris R, Baviera ME, et al. Diffusion and perfusion MR imaging in cases of Alzheimer's disease: correlations with cortical atrophy and lesion load. Am J Neuroradiol 2001; 22:1030-1036.

33 Kantarci K, Jack CR Jr, Xu YC, et al. Mild cognitive impairment and Alzheimer disease: regional diffusivity of water. Radiology 2001; 219:101-107.

34 Bozzali M, Franceschi M, Falini A, et al. Quantification of tissue damage in $A D$ using diffusion tensor and magnetization transfer MRI. Neurology 2001; 57:1135-1137.

35 Hanyu $\mathrm{H}$, Asano $\mathrm{T}$, Sakurai $\mathrm{H}$, et al. Magnetization transfer measurements of the hippocampus in the early diagnosis of Alzheimer's disease. J Neurol Sci 2001; 188:79-84.

36 Huang W, Alexander GE, Chang L, et al. Brain metabolite concentration and dementia severity in Alzheimer's disease: a (1)H MRS study. Neurology 2001; 57:626-632.

37 Valenzuela MJ, Sachdev P. Magnetic resonance spectroscopy in AD. Neurology 2001; 56:592-598.

38 Mielke $\mathrm{R}$, Schopphoff $\mathrm{HH}$, Kugel $\mathrm{H}$, et al. Relation between $1 \mathrm{H}$ MR spectroscopic imaging and regional cerebral glucose metabolism in Alzheimer's disease. Int J Neurosci 2001; 107:233-245.

A combination of multimodal functional brain data in AD.

39 Rose SE, Chen F, Chalk JB, et al. Loss of connectivity in Alzheimer's disease: an evaluation of white matter tract integrity with colour coded MR diffusion tensor imaging. J Neurol Neurosurg Psychiatry 2000; 69:528-530.

40 Mega MS, Cummings JL, O'Connor SM, et al. Cognitive and metabolic responses to metrifonate therapy in Alzheimer disease. Neuropsychiatry Neuropsychol Behav Neurol 2001; 14:63-68.

41 Potkin SG, Anand R, Fleming K, et al. Brain metabolic and clinical effects of rivastigmine in Alzheimer's disease. Int J Neuropsychopharmacol 2001; $4: 223-230$.

42 Nakano S, Asada T, Matsuda $\mathrm{H}$, et al. Donepezil hydrochloride preserves regional cerebral blood flow in patients with Alzheimer's disease. J Nucl Med 2001; 42:1441-1445.

43 Herholz K, Lercher M, Wienhard K, et al. PET measurement of cerebral acetylcholine esterase activity without blood sampling. Eur J Nucl Med 2001; 28:472-477.

This study demonstrates the use of functional imaging to assess the cholinergic hypothesis in AD.

44 Tanaka N, Fukushi $\mathrm{K}$, Shinotoh $\mathrm{H}$, et al. Positron emission tomographic measurement of brain acetylcholinesterase activity using $\mathrm{N}-[(11) \mathrm{C}]$ methylpiperidin-4-yl acetate without arterial blood sampling: methodology of shape analysis and its diagnostic power for Alzheimer's disease. J Cereb Blood Flow Metab 2001; 21:295-306.

This study demonstrates the use of functional imaging to assess the cholinergic hypothesis in AD.

45 Shinotoh $\mathrm{H}$, Aotsuka $\mathrm{A}$, Fukushi $\mathrm{K}$, et al. Effect of donepezil on brain acetylcholinesterase activity in patients with AD measured by PET. Neurology 2001; 56:408-410.

46 Cagnin A, Brooks DJ, Kennedy AM, et al. In-vivo measurement of activated microglia in dementia. Lancet 2001; 358:461-467.

Imaging microglial activation as a marker for the brain's immune response to degeneration in $A D$.

47 Arnaiz $\mathrm{E}$, Jelic $\mathrm{V}$, Almkvist $\mathrm{O}$, et al. Impaired cerebral glucose metabolism and cognitive functioning predict deterioration in mild cognitive impairment. Neuroreport 2001; 12:851-855.

48 Johnson KA, Jones K, Holman BL, et al. Preclinical prediction of Alzheimer's disease using SPECT. Neurology 1998; 50:1563-1571.
49 de Leon MJ, Convit A, Wolf OT, et al. Prediction of cognitive decline in normal

- elderly subjects with 2-[(18)F]fluoro-2-deoxy-D-glucose/positron-emission tomography (FDG/PET). Proc Natl Acad Sci U S A 2001; 98:10966-10971.

A major contribution using anatomically driven metabolic measurement in medial temporal structures. A metabolic reduction in the entorhinal cortex predicted cognitive impairment in healthy elderly people.

50 Cohen RA, Paul RH, Zawacki TM, et al. Single photon emission computed tomography, magnetic resonance imaging hyperintensity, and cognitive impairments in patients with vascular dementia. J Neuroimaging 2001; 11:253-260.

51 Minoshima S, Foster NL, Sima AA, et al. Alzheimer's disease versus dementia with Lewy bodies: cerebral metabolic distinction with autopsy confirmation. Ann Neurol 2001; 50:358-365.

A comparison of cerebral metabolism in patients with a definite diagnosis.

52 Okamura N, Arai H, Higuchi M, et al. [18F]FDG-PET study in dementia with

- Lewy bodies and Alzheimer's disease. Prog Neuropsychopharmacol Bio Psychiatry 2001; 25:447-456.

This study shows impaired brain activity in two siblings and a population with DLB.

53 Cordery RJ, Tyrrell PJ, Lantos PL, Rossor MN. Dementia with Lewy bodies studied with positron emission tomography. Arch Neurol 2001; 58:505-508.

54 Imamura $\mathrm{T}$, Ishii $\mathrm{K}$, Hirono $\mathrm{N}$, et al. Occipital glucose metabolism in dementia with Lewy bodies with and without Parkinsonism: a study using positron emission tomography. Dement Geriatr Cogn Disord 2001; 12:194-197.

55 Hisanaka $\mathrm{K}$, Suzuki $\mathrm{H}$, Tanji $\mathrm{H}$, et al. Fluoro-DOPA and FDG positron emission tomography in a case of pathologically verified pure diffuse Lewy body disease. J Neurol 2001; 248:905-906.

$56 \mathrm{Hu}$ XS, Okamura N, Arai $\mathrm{H}$, et al. 18F-fluorodopa PET study of striatal dopamine uptake in the diagnosis of dementia with Lewy bodies. Neurology 2000; 55:1575-1577.

57 Barber R, Panikkar A, McKeith IG. Dementia with Lewy bodies: diagnosis and management. Int J Geriatr Psychiatry 2001; 16 (Suppl 1):S12-S18.

58 Lobotesis K, Fenwick JD, Phipps A, et al. Occipital hypoperfusion on SPECT in dementia with Lewy bodies but not AD. Neurology 2001; 56:643-649.

59 Barber R, McKeith IG, Ballard C, et al. A comparison of medial and lateral temporal lobe atrophy in dementia with Lewy bodies and Alzheimer's disease: magnetic resonance imaging volumetric study. Dement Geriatr Cogn Disord 2001; 12:198-205.

60 Berding G, Odin P, Brooks DJ, et al. Resting regional cerebral glucose metabolism in advanced Parkinson's disease studied in the off and on conditions with [(18)F]FDG-PET. Mov Disord 2001; 16:1014-1022.

61 Brito-Marques PR, Mello RV, Montenegro L. Classic Pick's disease type with ubiquitin-positive and tau-negative inclusions: case report. Arq Neuropsiquiatr 2001; 59:128-133.

62 Portet F, Cadilhac C, Touchon J, Camu W. Cognitive impairment in motor neuron disease with bulbar onset. Amyotroph Lateral Scler Other Motor Neuron Disord 2001; 2:23-29.

63 de Vries PJ, Honer WG, Kemp PM, McKenna PJ. Dementia as a complication of schizophrenia. J Neurol Neurosurg Psychiatry 2001; 70:588-596.

64 Pagani M, Salmaso D, Ramstrom C, et al. Mapping pathological (99m)Tc-d,I-

- hexamethylpropylene amine oxime uptake in Alzheimer's disease and frontal lobe dementia with SPECT. Dement Geriatr Cogn Disord 2001; 12:177-184.

An analysis of images standardized in a tridimensional space.

65 Garraux G, Salmon E, Degueldre C, et al. Comparison of impaired subcortico-frontal metabolic networks in normal aging, subcortico-frontal dementia, and cortical frontal dementia. Neurolmage 1999; 10:149-162.

66 Sarazin M, Pillon B, Giannakopoulos P, et al. Clinicometabolic dissociation of cognitive functions and social behavior in frontal lobe lesions. Neurology 1998; 51:142-148

67 Miller BL, Seeley WW, Mychack P, et al. Neuroanatomy of the self: evidence from patients with frontotemporal dementia. Neurology 2001; 57:817-821.

68 Martin JA, Craft DK, Su JH, et al. Astrocytes degenerate in frontotemporal dementia: possible relation to hypoperfusion. Neurobiol Aging 2001; 22:195207.

69 Mathews D, Unwin DH. Quantitative cerebral blood flow imaging in a patient with the Heidenhain variant of Creutzfeldt-Jakob disease. Clin Nucl Med 2001; 26:770-773.

70 Peigneux P, Salmon E, Garraux G, et al. Neural and cognitive bases of upper - limb apraxia in corticobasal degeneration. Neurology 2001; 57:1259-1268. A cognitive model is used to explore the neural substrate of apraxia in patients with CBD. 